\title{
Risk factors for Staphylococcus aureus nasal colonization in Danish middle-aged and elderly twins
}

\author{
P. S. Andersen - L. A. Larsen • V. G. Fowler Jr • \\ M. Stegger $\cdot$ R. L. Skov $\cdot$ K. Christensen
}

Received: 19 February 2013 / Accepted: 9 April 2013 /Published online: 9 May 2013

(C) The Author(s) 2013. This article is published with open access at Springerlink.com

\begin{abstract}
Staphylococcus aureus is a human commensal bacterium found in the nasal cavity and other body sites. Identifying risk factors for $S$. aureus nasal carriage is of interest, as nasal carriage is a risk factor for subsequent invasive infection. We recently investigated the influence of host genetics on S. aureus carriage in Danish middle-aged and elderly twins, which indicated no significant heritability that could account for the observed $S$. aureus carriage. In the present study, we performed a questionnaire-based study of S. aureus colonization on the same cohort of 2,196 Danish middle-aged and elderly twins to identify specific risk factors for $S$. aureus nasal colonization, including analyzing the paired twins $(n=478)$ that were discordant for $S$. aureus colonization. We found associations between risk factors and $S$. aureus nasal colonization among middle-aged and elderly twins, including age, male gender, psoriasis, and atopic diseases. Also, present living on a farm is clearly associated with $S$. aureus colonization, while smoking had a borderline statistically significant protective effect.
\end{abstract}

Electronic supplementary material The online version of this article (doi:10.1007/s10096-013-1882-0) contains supplementary material, which is available to authorized users.

P. S. Andersen $(\bowtie) \cdot$ M. Stegger $\cdot$ R. L. Skov

Microbiology Research and Infection Control, Statens Serum

Institut, Copenhagen, Denmark

e-mail:psa@ssi.dk

L. A. Larsen $\cdot$ K. Christensen

The Danish Twin Registry, Institute of Public Health, University of Southern Denmark, Odense, Denmark

V. G. Fowler Jr

Duke Medical Center, Duke University, Durham, NC, USA

K. Christensen

Department of Clinical Genetics and Department of Clinical

Biochemistry and Pharmacology, Odense University Hospital,

Odense, Denmark

\section{Introduction}

Staphylococcus aureus is an opportunistic pathogen that normally is found as a commensal of the human skin, as well as in the nasal cavity. In cross-sectional studies, about $20-40 \%$ of the adult population is colonized with $S$. aureus [1-5]. Three types of carriage have been described: permanent carriage, i.e., carriage over a prolonged period of time and most often with the same strain (approximately $20 \%$ ), intermittent carriage (approximately $30 \%$ ), and noncarriage $(50 \%)[2,6]$. These may be classified as only two groups, carriers and non-carriers [7], as the intermittent carriage and non-carriage groups are similar in respect to the risk of acquiring $S$. aureus infection. We recently reported that the $S$. aureus carriage rate in a large cohort of elderly Danish twins was $24.7 \%$ and was not primarily due to genetic factors [8]. However, identifying risk factors for $S$. aureus carriage is of major concern, as nasal carriage is a significant risk factor for subsequent invasive infection $[6,9]$.

In the present study, we used a large Danish twins cohort to examine potential associations between putative risk factors and $S$. aureus nasal colonization. We investigate the cohort both as individuals and also by pairing the twins, taking into account that their upbringings have been similar.

\section{Materials and methods}

Study population

The Danish Twin Registry is a nationwide, population-based registry established in 1954 containing data of $>85,000$ twin pairs born between 1870 and 2009. In the period 2008-2011, twins born in Denmark between 1931 and 1969 were invited to a clinical investigation in one of five centers in Denmark. Nasal swabs for the determination of the presence of $S$. aureus in the nasal bacterial flora were collected, resulting in a total of 
2,213 nasal swabs. The study was approved by the Science Ethics Committee for Southern Denmark (project number SVF-19980072, addendum nos. 8 and 9) with appropriate informed consent following the guidelines of the approved protocol $[8,10]$.

\section{Determination of $S$. aureus colonization in the anterior nares}

Colonization was determined by isolating $S$. aureus from nasal swabs, as previously described [8]. Briefly, the anterior nares were swabbed using E-swabs (Copan, Italy) and transferred directly to carrier medium. S. aureus-positive swabs were determined by culturing on $S$. aureus-selective plates (chromID, bioMérieux, France) for $48 \mathrm{~h}$ and positive colonies were identified by colony morphology and catalase test (SSI Diagnostika, Denmark).

\section{Epidemiological data}

The twins in the study were asked to fill out two questionnaires. One concerned diseases, infections, environment, and contact with animals which were variables expected to be associated with the carriage of $S$. aureus. The other questionnaire was about general health. Based on these questionnaires, the preselected covariates for the analyses included psoriasis, atopic dermatitis, asthma, allergy, (repeated) staphylococcal infection, hospitalization within 3 months, smoking, urban/rural living at the age of 6 years, animals in the household (animals), and present living or working at a farm. All covariates were binary. Gender and age (below and above the median age of subjects) were also covariates in this study. All covariates were tested for pairwise correlation in order to avoid collinear covariates.

To obtain sufficiently high numbers, the answers to questions regarding atopic dermatitis, asthma, and allergy were merged into "atopic disease". The study included 2,213 twins, among which 2,196 (99.2\%) had filled out the questionnaires. The frequency of replies missing was, on average, $1.4 \%$ and, for all covariates, less than $4.6 \%$. The 2,196 twins comprised 627 intact twin pairs $(1,254$ twins who both participated) and 942 broken pairs (where only one twin from the pair participated). Dismissing twins with unknown zygosity, a separate twin analysis was made based on the 239 discordant twin pairs in which one of the twins in the twin pair was a carrier of $S$. aureus. This sub-analysis comprised 40 monozygotic (MZ), 112 same-sex dizygotic (SS-DZ), and 87 opposite-sex dizygotic (OS-DZ) twin pairs. The twins $(N=2,196)$ had a mean age of $62.6( \pm 7.5)$ years and consisted of $51.2 \%$ males. The age distributions among men and women were similar, with $49.5 \%$ of the men aged $<63$ years and $47.7 \%$ of the women aged $<63$ years. Among the discordant twin pairs, $50 \%$ were males. The age distribution was more skewed than the overall sample, with $33.1 \%$ of the men aged $<63$ years and $40.2 \%$ of the women aged $<63$ years.

Statistical methods

The analyses were performed in two steps: first for all participants disregarding that the participants were twins and second for discordant twin pairs alone, where one of the twins in a pair was colonized with $S$. aureus and the other was not. All analyses were performed using the statistical program Stata 11.2.

In the univariate analyses of covariates, logistic regression was used, including the analyses when controlling for age and gender. The correlation between covariates was assessed using tetrachoric correlations.

For the discordant twin pairs, a conditional multivariate logistic regression was made, modeling for fixed effects by grouping the twins pairwise. If one twin did not answer a question, the answer from the co-twin was removed as well. Conditional logistic regression was made for modeling fixed effects, and the twins were grouped by their pair number. The same covariates were included, except for age, which was matched for each twin pair by definition.

\section{Results}

Prevalence by age group and gender

Based on the overall sample $(N=2,196)$ the overall prevalence of $S$. aureus nasal colonization was $24.7 \%$ [95\% confidence interval (CI): $22.9 \%-26.5 \%$ ]. Males were significantly more likely to be colonizers than females [males $27.6 \%$ (95\% CI: $25.0 \%-30.3 \%$ ) vs. females $21.6 \%$ (95\% CI: $19.1 \%-24.0 \%), p<0.01]$. Also, the prevalence of $S$. aureus nasal colonization in the younger half of the individuals did not differ significantly from that in the older half ( $26.0 \%$ vs. $23.3 \% ; p=0.15$ ). Among discordant twin pairs, males were also significantly more likely to be colonized than females [56.1\% (95\% CI: $49.7 \%-62.4 \%$ ) vs. $43.9 \%$ (95 \% CI: $37.6 \%-50.3 \%$ ), $p<0.01]$. Here, the prevalence by age was almost the same, the youngest being $49.8 \%$ (95\% CI: $44.2 \%-55.5 \%$ ), and the oldest being $50.3 \%$ (95 \% CI: $42.8 \%-57.8 \%)$.

Univariate analyses

Colonization of $S$. aureus was tested in univariate analyses for all the selected risk factors, including an analysis adjusting for age and gender. Psoriasis, atopic disease, living/working at a farm, and animals in the household were significant. Living/working at a farm doubles the risk (odds ratio (OR) 2.05; $95 \% \mathrm{CI}: 1.35-3.11$ ), while the risk 
increased by $73 \%$ for those having psoriasis (OR 1.73; $95 \%$ CI: 1.16-2.58) and having atopic disease increased the risk by $36 \%$ (OR $1.36 ; 95 \%$ CI:1.11-1.68) of S. aureus colonization. Adjusting for age and gender made "animals in the household" not significant (OR 1.20; 95 \% CI:0.97-1.48), while being a current smoker was borderline significant (OR 0.78; $95 \%$ CI:0.60-1.01). Generally, adjusting for age and gender did not change the OR notably (Table 1).

When analyzing the discordant twin pairs, only gender, psoriasis, and living at a farm were statistically significant, the last two with large confidence limits (Supplementary Table 1).

Multivariate logistic regression analyses

For the overall cohort, a multivariate logistic regression analysis was made including all risk factors listed in Table 1, except "ever smoked" and repeated S. aureus infection, since they were highly correlated with, respectively, "current smoker" and $S$. aureus infection, leaving $N=2,033$ for analyses (Table 2). In the multivariate analyses, gender, psoriasis, atopic disease, current smoker, and living/working at a farm were statistically significant, while age, $S$. aureus infection, urban/rural living at the age of 6 years, animals in the household, and hospitalization within the last 3 months were not.

Omitting the statistically non-significant risk factors, except for age, from the analysis made smoking borderline statistically significant $(p=0.05)(\mathrm{OR} 0.76 ; 95 \%$ CI:0.58-
1.00) (Table 3). The largest effects were seen for living/working on a farm (OR2.09; $95 \%$ CI:1.36-3.21) and psoriasis (OR=1.82; $95 \% \mathrm{CI}: 1.21-2.74)$.

Conditional logistic regression for discordant twin pairs

The same selected risk factors (shown in Table 3) were included in the analysis for the discordant twin pairs, except for age, which was perfectly matched for each twin pair $(N=436)$ (Table 4$)$. In this sub-analysis, gender, psoriasis (OR 5.14; $95 \%$ CI:1.81-14.59) and living/working at a farm (OR 4.82; $95 \%$ CI: 1.40 16.60) were significant, but with large confidence limits, while current smoker was borderline significant and atopic disease was no longer significant.

This analysis was also performed for same-sexed discordant twin pairs alone $(N=280)$, where the covariate gender also was omitted. This analysis showed that psoriasis, atopic diseases, and living/working at a farm were significant, while "current smoker" was not significant (table not shown).

\section{Discussion}

As genetic factors were recently shown to be of limited importance for $S$. aureus nasal colonization [8], here, we aimed to identify specific risk factors that could improve our

Table 1 Univariate analyses of risk factors in the overall cohort

\begin{tabular}{|c|c|c|c|c|c|c|c|c|c|c|}
\hline Risk factor & Exposure & $N^{\mathrm{a}}$ & $\begin{array}{l}\text { Colonized, } \\
n(\%)\end{array}$ & $\begin{array}{l}\text { Not colonized, } \\
n(\%)\end{array}$ & $\mathrm{OR}^{\mathrm{b}}$ & $95 \% \mathrm{CI}$ & $p$-Value ${ }^{\mathrm{c}}$ & OR adjusted ${ }^{\mathrm{b}}$ & $95 \% \mathrm{CI}$ & $p$-Value ${ }^{\mathrm{c}}$ \\
\hline Total number & & 2,196 & $542(24.7)$ & $1,654(75.3)$ & & & & & & \\
\hline Age group & $\geq 63$ & 2,196 & $249(45.9)$ & $819(49.5)$ & 0.87 & $(0.71-1.05)$ & 0.1 & & & \\
\hline Gender & Females & 2,196 & $231(42.6)$ & $840(50.8)$ & 0.72 & $(0.59-0.88)$ & $<0.01$ & & & \\
\hline Psoriasis & Yes & 2,097 & $40(7.7)$ & $72(4.6)$ & 1.73 & $(1.16-2.58)$ & $<0.01$ & 1.76 & $(1.17-2.62)$ & $<0.01$ \\
\hline Atopic disease $^{\mathrm{d}}$ & Yes & 2,196 & $176(32.5)$ & $431(26.1)$ & 1.36 & $(1.11-1.68)$ & $<0.01$ & 1.42 & $(1.15-1.76)$ & $<0.01$ \\
\hline S. aureus infection & Yes & 2,175 & $25(4.7)$ & $86(5.3)$ & 0.88 & $(0.56-1.39)$ & 0.6 & 0.89 & $(0.56-1.40)$ & 0.6 \\
\hline S. aureus infection & Yes, repeat & 2,175 & $10(1.9)$ & $20(1.2)$ & 1.5 & $(0.71-3.28)$ & 0.3 & 1.60 & $(0.74-3.45)$ & 0.2 \\
\hline Current smoker & Yes & 2,183 & $89(16.5)$ & $325(19.8)$ & 0.8 & $(0.62-1.03)$ & 0.1 & 0.78 & $(0.60-1.01)$ & $<0.06$ \\
\hline Ever smoked & Yes & 2,183 & $310(57.8)$ & $971(59.0)$ & 0.95 & $(0.78-1.16)$ & 0.6 & 0.94 & $(0.77-1.14)$ & 0.5 \\
\hline Childhood, 6 years ${ }^{\mathrm{e}}$ & Urban & 2,186 & $155(28.7)$ & $468(28.5)$ & 1.01 & $(0.81-1.25)$ & 0.9 & 1.01 & $(0.81-1.25)$ & 1.0 \\
\hline Farm living/working & Yes & 2,174 & $396^{\mathrm{f}}(7.3)$ & $60^{f}(3.7)$ & 2.05 & $(1.35-3.11)$ & $<0.01$ & 1.97 & $(1.30-3.00)$ & $<0.01$ \\
\hline Animals & Yes & 2,160 & $195(36.7)$ & $516(31.7)$ & 1.25 & $(1.02-1.54)$ & $<0.04$ & 1.20 & $(0.97-1.48)$ & $<0.09$ \\
\hline Hospitalization $^{\mathrm{g}}$ & Yes & 2,174 & $17(3.2)$ & $63(3.9)$ & 0.81 & $(0.47-1.40)$ & 0.5 & 0.80 & $(0.46-1.38)$ & 0.4 \\
\hline
\end{tabular}

\footnotetext{
${ }^{\text {a }}$ Varying numbers due to missing values

${ }^{\mathrm{b}} \mathrm{OR}$ and $p$-values are shown with and without adjusting for age and gender

${ }^{\mathrm{c}}$ Logistic regression

${ }^{\mathrm{d}}$ Includes asthma, atopic dermatitis, and allergy

${ }^{\mathrm{e}}$ Urban/rural living when 6 years old

${ }^{\mathrm{f}}$ In eight cases, there were pigs on the farm resulting in $1.5 \%$ for the colonized and $0.5 \%$ for the non-colonized $(\mathrm{p}<0.05)$

${ }^{\mathrm{g}}$ Hospitalization within the last 3 months
} 
Table 2 Multivariate analyses of risk factors in the overall cohort $(N=$ $2,033)^{\mathrm{a}}$

\begin{tabular}{|c|c|c|c|c|}
\hline Risk factor & Exposure & OR & $95 \% \mathrm{CI}$ & $p$-Value \\
\hline Age group & $\geq 63$ & 0.87 & $(0.71-1.08)$ & 0.21 \\
\hline Gender & Female & 0.67 & $(0.54-0.82)$ & $<0.01$ \\
\hline Psoriasis & Yes & 1.81 & $(1.20-2.73)$ & $<0.01$ \\
\hline Atopic disease $^{\mathrm{b}}$ & Yes & 1.45 & $(1.16-1.82)$ & $<0.01$ \\
\hline S. aureus infection & Yes & 0.86 & $(0.53-1.41)$ & 0.56 \\
\hline Current smoker & Yes & 0.75 & $(0.57-0.99)$ & $<0.05$ \\
\hline Childhood, 6 years ${ }^{\mathrm{c}}$ & Urban & 1.02 & $(0.81-1.28)$ & 0.89 \\
\hline Farm living/working & Yes & 1.90 & $(1.20-3.00)$ & $<0.01$ \\
\hline Animals & Yes & 1.14 & $(0.91-1.44)$ & 0.26 \\
\hline Hospitalization $^{\mathrm{d}}$ & Yes & 0.82 & $(0.46-1.45)$ & 0.50 \\
\hline
\end{tabular}

a 2,033 twins have answered all the selected questions

${ }^{\mathrm{b}}$ Includes asthma, atopic dermatitis, and allergy

${ }^{\mathrm{c}}$ Urban/rural living when 6 years old

${ }^{\mathrm{d}}$ Hospitalization within the last 3 months

understanding of factors associated with $S$. aureus nasal colonization using a large twin population.

We have investigated the cohort both as individuals and as twin pairs. We showed consistently that living on a farm doubles the risk for $S$. aureus nasal colonization, which has not previously been demonstrated. Also, we saw a similar correlation between psoriasis and colonization. This is in agreement with two studies that found an association between psoriasis and $S$. aureus infection and nasal colonization [11, 12]. The less apparent, although significant, association to atopic disease is in agreement with previous studies, as atopic dermatitis patients have a high incidence of $S$. aureus infections and increased rates of nasal carriage [13]. Smoking appears to be antagonistic to $S$. aureus nasal colonization, which is in agreement with previous studies [14-16]. Recent hospitalization was not associated with S. aureus colonization, in contrast to a recent study [1]; however, the sample size used was not large enough to rule out a possible association.

Table 3 Multivariate analyses of selected risk factors in the overall cohort $(N=2,073)^{\mathrm{a}}$

\begin{tabular}{llllr}
\hline Risk factor & Exposure & OR & $95 \%$ CI & $p$-Value \\
\hline Age group & $\geq 63$ & 0.86 & $(0.70-1.05)$ & 0.15 \\
Gender & Female & 0.68 & $(0.56-0.84)$ & $<0.01$ \\
Psoriasis & Yes & 1.82 & $(1.21-2.74)$ & $<0.01$ \\
Atopic disease & Yes & 1.43 & $(1.15-1.79)$ & $<0.01$ \\
Current smoker & Yes & 0.76 & $(0.58-1.00)$ & 0.05 \\
Farm living/working & Yes & 2.09 & $(1.36-3.21)$ & $<0.01$ \\
\hline
\end{tabular}

${ }^{a} 2,073$ twins have answered all the selected questions

${ }^{\mathrm{b}}$ Includes asthma, atopic dermatitis, and allergy
Table 4 Multivariate analysis of selected risk factors among twins discordant for Staphylococcus aureus colonization $(N=436)^{\mathrm{a}}$

\begin{tabular}{llllr}
\hline Risk factor & Exposure & OR & $95 \%$ CI & $p$-Value \\
\hline Gender & Female & 0.51 & $(0.31-0.86)$ & 0.01 \\
Psoriasis & Yes & 5.14 & $(1.81-14.59)$ & $<0.01$ \\
Atopic disease $^{b}$ & Yes & 1.52 & $(0.95-2.46)$ & $<0.09$ \\
Current smoker & Yes & 0.59 & $(0.34-1.02)$ & 0.06 \\
Farm living/working & Yes & 4.82 & $(1.40-16.60)$ & $<0.02$ \\
\hline
\end{tabular}

${ }^{\mathrm{a}} 436$ discordant twin pairs have answered all the selected questions

${ }^{\mathrm{b}}$ Includes asthma, atopic dermatitis, and allergy

Rural living as a child was not associated with $S$. aureus colonization but current living/working on a farm was associated with $S$. aureus nasal colonization, suggesting that these individuals may be colonized or transiently contaminated from the animals on the farm, as previously described [17]. This may be part of the reason why we find that two of the $S$. aureus colonizers were positive for CC398 (data not shown), a lineage normally associated with pigs [18], which is in agreement with the finding that, among the $S$. aureus colonizers and those not colonized with $S$. aureus, there were eight individuals in each group that worked or lived on a farm with pigs. Further samplings of these individuals living/working on farms could clarify this observation despite the non-colonized group being approximately three times bigger $(\mathrm{P}<0.05)$.

Furthermore, we also address the same questions taking into consideration that it is a twin study and we can, therefore, adjust for common childhood environment and common genes. Although the numbers are greatly reduced, the intrapair analysis confirmed the findings. Thus, the significant association between nasal colonization and living on a farm as well as psoriasis are retained. With this further matching, smoking and atopic disease association became significant.

The current study only investigates the association between specific risk factors and a single time point of $S$. aureus nasal colonization. Therefore, this study does not address the issue of association between environmental conditions and persistent $S$. aureus or non-colonization, where the association may be different. However, certain factors indicate that such a grouping may only strengthen the observed associations. A recent study of $S$. aureus nasal colonization among Norwegian adults where there had been more than one sampling of the studied individuals performed clearly showed that smoking was a protective factor in relation to $S$. aureus nasal carriage [14]. In our study, we observed a borderline association. No other associations of risk factors and S. aureus nasal colonization coincide in the two studies. Olsen and coworkers showed that an inverse correlation exists between vitamin D levels and $S$. aureus colonization [14]. 
Atopic disease, including atopic dermatitis, allergies, and asthma, was also weakly associated with $S$. aureus nasal colonization. The need to converge all three questions will certainly reduce the power of any specific association, as we would expect there to be an association with atopic dermatitis but not necessarily asthma or the less well defined condition of allergies. The sample size was too small to investigate the association between atopic dermatitis and $S$. aureus colonization.

The questionnaire has some limitations due to the types of questions it contained. We did not clearly distinguish between farm animals and pets and, therefore, had to group them as one. The disease questions are also, to a certain extent, difficult to answer, as some people may not know the correct terminology. This is the case for atopic dermatitis, asthma, and allergies and, to a lesser degree, psoriasis. This misclassification is unlikely to be associated with $S$. aureus nasal colonization and, therefore, the misclassification will tend to underestimate the association between these risk factors and $S$. aureus colonization.

\section{Conclusion}

In conclusion, the strongest risk factors of Staphylococcus aureus colonization were male gender, living on a farm, psoriasis, and any atopic disease, while smoking had a borderline protective effect. Future studies involving the closer environment of $S$. aureus, the microbial community of the nares, may give further clues as to the selective colonization of $S$. aureus in only a fraction of all individuals.

Acknowledgments V.G. Fowler Jr was supported by the National Institutes of Health (R01-AI068804). The Danish Twin Registry is supported by a grant from the National Programme for Research Infrastructures 2007 from the Danish Agency for Science Technology and Innovation.

Conflict of interest The authors declare that they have no conflict of interest.

Open Access This article is distributed under the terms of the Creative Commons Attribution License which permits any use, distribution, and reproduction in any medium, provided the original author(s) and the source are credited.

\section{References}

1. Gorwitz RJ, Kruszon-Moran D, McAllister SK, Mcquillan G, McDougal LK, Fosheim GE et al (2008) Changes in the prevalence of nasal colonization with Staphylococcus aureus in the United States, 2001-2004. J Infect Dis 197:1226-1234. doi:10.1086/ 533494, http://www.ncbi.nlm.nih.gov/pubmed/18422434

2. Kuehnert MJ, Kruszon-Moran D, Hill HA, McQuillan G, McAllister SK, Fosheim G et al (2006) Prevalence of Staphylococcus aureus nasal colonization in the United States, 2001-2002. J Infect Dis
193:172-179. doi:10.1086/499632, http://www.ncbi.nlm.nih.gov/ pubmed/16362880

3. Ruimy R, Angebault C, Djossou F, Dupont C, Epelboin L, Jarraud $S$ et al (2010) Are host genetics the predominant determinant of persistent nasal Staphylococcus aureus carriage in humans? J Infect Dis 202:924-934. doi:10.1086/655901, http:// www.ncbi.nlm.nih.gov/pubmed/20677941

4. Wertheim HFL, Melles DC, Vos MC, van Leeuwen W, van Belkum A, Verbrugh HA et al (2005) The role of nasal carriage in Staphylococcus aureus infections. Lancet Infect Dis 5:751-762. doi:10.1016/S1473-3099(05)70295-4, http://www.ncbi.nlm.nih.gov/ pubmed/16310147

5. Ruimy R, Armand-Lefevre L, Barbier F, Ruppé E, Cocojaru R, Mesli Y et al (2009) Comparisons between geographically diverse samples of carried Staphylococcus aureus. J Bacteriol 191:5577-83. doi:10.1128/ JB.00493-09, http://www.pubmedcentral.nih.gov/articlerender.fcgi? artid $=2737961 \&$ tool $=$ pmcentrez\&rendertype $=$ abstract

6. Wertheim HFL, Vos MC, Ott A, van Belkum A, Voss A, Kluytmans JAJW et al (2004) Risk and outcome of nosocomial Staphylococcus aureus bacteraemia in nasal carriers versus noncarriers. Lancet 364:703-705. doi:10.1016/S0140-6736(04) 16897-9, http://www.ncbi.nlm.nih.gov/pubmed/15325835

7. van Belkum A, Verkaik NJ, de Vogel CP, Boelens HA, Verveer J, Nouwen JL et al (2009) Reclassification of Staphylococcus aureus nasal carriage types. J Infect Dis 99:1820-1826. doi:10.1086/ 599119, http://www.ncbi.nlm.nih.gov/pubmed/19419332

8. Andersen PS, Pedersen JK, Fode P, Skov RL, Fowler VG Jr, Stegger $M$ et al (2012) Influence of host genetics and environment on nasal carriage of Staphylococcus aureus in Danish middle-aged and elderly twins. J Infect Dis 206:1178-1184. doi:10.1093/infdis/ jis491, http://www.pubmedcentral.nih.gov/articlerender.fcgi? artid $=3448969$ \& tool $=$ pmcentrez\&rendertype $=$ abstract

9. von Eiff C, Becker K, Machka K, Stammer H, Peters G (2001) Nasal carriage as a source of Staphylococcus aureus bacteremia. Study Group. N Engl J Med 344:11-16

10. Skytthe A, Christiansen L, Kyvik KO, Bødker FL, Hvidberg L, Petersen I et al (2013) The Danish Twin Registry: linking surveys, national registers, and biological information. Twin Res Hum Genet 16(1):104-111. doi:10.1017/thg.2012.77, http://www.ncbi.nlm.nih.gov/pubmed/23084092

11. Tomi NS, Kränke B, Aberer E (2005) Staphylococcal toxins in patients with psoriasis, atopic dermatitis, and erythroderma, and in healthy control subjects. J Am Acad Dermatol 53:67-72. doi:10.1016/j.jaad.2005.02.034

12. Balci DD, Duran N, Ozer B, Gunesacar R, Onlen Y, Yenin JZ (2009) High prevalence of Staphylococcus aureus cultivation and superantigen production in patients with psoriasis. Eur J Dermatol 19:238-242. doi:10.1684/ejd.2009.0663, http:// www.ncbi.nlm.nih.gov/pubmed/19286488

13. Breuer K, Haussler S, Kapp A, Werfel T (2002) Staphylococcus aureus: colonizing features and influence of an antibacterial treatment in adults with atopic dermatitis. Br J Dermatol 147:55-61

14. Olsen K, Falch BM, Danielsen K, Johannessen M, Ericson Sollid JU, Thune I et al (2012) Staphylococcus aureus nasal carriage is associated with serum 25-hydroxyvitamin D levels, gender and smoking status. The Tromsø Staph and Skin Study. Eur J Clin Microbiol Infect Dis 31:465-473. doi:10.1007/s10096-011-1331$\mathrm{x}$, http://www.pubmedcentral.nih.gov/articlerender.fcgi?artid= $3303067 \&$ tool $=$ pmcentrez\&rendertype $=$ abstract

15. Wang J-T, Liao C-H, Fang C-T, Chie W-C, Lai M-S, Lauderdale T-L et al (2009) Prevalence of and risk factors for colonization by methicillinresistant Staphylococcus aureus among adults in community settings in Taiwan. J Clin Microbiol 47:2957-2963. doi:10.1128/JCM.0085309, http://www.pubmedcentral.nih.gov/articlerender.fcgi?artid= 2738089\&tool=pmcentrez\&rendertype $=$ abstract 
16. Melles DC, Pauw E, Van den Boogaard L, Boelens HM, Peters J, Peeters JK et al (2008) Host-microbe interplay in persistent Staphylococcus aureus nasal carriage in HIV patients. Microbes Infect 10:151-158. doi:10.1016/ j.micinf.2007.10.017, http://www.ncbi.nlm.nih.gov/pubmed/ 18248760

17. Aubry-Damon H, Grenet K, Sall-Ndiaye P, Che D, Cordeiro E, Bougnoux M-E et al (2004) Antimicrobial resistance in commensal flora of pig farmers. Emerg Infect Dis 10:873-879. doi:10.3201/eid1005.030735, http://www.pubmedcentral.nih.gov/ articlerender.fcgi? artid $=3323198 \&$ tool=pmcentrez\&rendertype $=$ abstract

18. Lewis HC, Mølbak K, Reese C, Aarestrup FM, Selchau M, Sørum M et al (2008) Pigs as source of methicillin-resistant Staphylococcus aureus CC398 infections in humans, Denmark. Emerg Infect Dis 14:1383-1389. doi:10.3201/eid1409.071576, http://www.pubmedcentral.nih.gov/articlerender.fcgi?artid= 2603104\&tool=pmcentrez\&rendertype $=$ abstract 